\title{
PlantaCiOnes FORESTALES VS. REGENERACIÓN NATURAL IN SITU: EL CASO DE LOS PINOS Y LA REHABILITACIÓN en el Parque Nacional Cofre de Perote
}

\author{
José Antonio Pensado-Fernández, Lázaro Rafael Sánchez-Velásquez ${ }^{1}$, Ma. del Rosario \\ Pineda-López y Francisco Díaz-Fleischer \\ Instituto de Biotecnología y Ecología Aplicada, Universidad Veracruzana, Xalapa, Veracruz, México. \\ ${ }^{1}$ Autor para la correspondencia: lasanchez@uv.mx
}

\begin{abstract}
Resumen: El conocimiento de la estructura y dinámica de la vegetación como estimadores del desempeño de las plantaciones forestales y de la regeneración natural en la rehabilitación es escaso, y tampoco se conoce el efecto de la ganadería extensiva sobre ambas. El objetivo de este estudio fue comparar el número de individuos por hectárea y la tasa anual de crecimiento entre las comunidades de pinos (Pinus patula, P. montezumae y P. teocote) procedentes de las plantaciones forestales y de la regeneración natural en sitios con dos diferentes condiciones (con y sin exclusión de ganado). Se encontraron diferencias significativas $(P<$ 0.0001) en la tasa anual de crecimiento de la altura, en el diámetro basal del tallo y en el diámetro promedio de cobertura entre las plantaciones vs. la regeneración natural y entre las dos condiciones de exclusión de ganado. El número de individuos establecidos por hectárea fue significativamente mayor en la regeneración natural con exclusión de ganado $(1,380 \pm 120$ y 1,130 \pm 130$)$ que en aquella sin exclusión $(430 \pm 250)$, y en las plantaciones forestales con o sin exclusión de ganado ( $995 \pm 90$ y $455 \pm 125$, respectivamente; $P<0.0001)$. Se concluye que la regeneración natural de pinos es una opción adecuada para la rehabilitación ecológica en terrenos degradados, especialmente si están protegidos del ganado, en comparación con las plantaciones forestales.
\end{abstract}

Palabras clave: exclusión de ganado, plantaciones forestales, regeneración natural, rehabilitación ecológica, tasa de crecimiento.

\begin{abstract}
There is limited knowledge regarding vegetation structure and dynamics as estimators of performance of forest plantations and natural regeneration in the rehabilitation of degraded lands, and neither is well estimated the effect of extensive livestock grazing on both. The objective of this study was to compare the number of individuals and mean growth rates amongst communities of pines (Pinus patula, P. montezumae, and P. teocote) established from plantation and from natural regeneration in two different conditions of reforestation (protected and not protected from livestock). Statistically significant differences $(P<0.0001)$ were found in annual growth rates in height, basal stem diameter and cover diameter amongst plantations and natural regeneration in sites with and without livestock exclusion. The number of individuals per hectare set was significantly higher in the natural regeneration under cattle exclusion $(1,380 \pm 120$ and $1,130 \pm 130)$ than without exclusion of livestock $(430 \pm 250)$ and forest plantations with or without exclusion of livestock $(995 \pm 90$ and $455 \pm 125$, respectively; $P<0.0001)$. Results suggest that natural regeneration of pines is an efficient alternative to forest plantations for the ecological rehabilitation of degraded lands, especially if protected from livestock.
\end{abstract}

Keywords: ecological rehabilitation, forest plantations, growth rate, livestock exclusion, natural regeneration.

E n las últimas dos décadas, la superficie de bosques en México ha disminuido a una tasa anual cercana al $0.37 \%$; para el 2010 se estimó que la superficie forestal representaba el 33\% de la superficie terrestre nacional (FAO, 2010, 2011). Esto plantea la necesidad de desarrollar nuevas estrategias encaminadas a la conservación de los fragmentos forestales remanentes y a la rehabilitación de los terrenos con aptitud forestal degradados.
Las plantaciones forestales, además de ser la opción más utilizada para satisfacer la demanda de madera como materia prima (Sedjo, 1999), han adquirido importancia como estrategia de rehabilitación de terrenos degradados (Michon y de Foresta, 1996; Lamb et al., 2005; Montagnini et al., 2004). Del 2005 al 2010 se ha reportado, en México, un incremento en la superficie de plantaciones forestales del $1.6 \%$ al $5 \%$ de la superficie forestal total nacional (FAO, 
2010, 2011). Si se considera la creciente atención a los programas de reforestación y la continua pérdida de bosques naturales, es de esperarse que estas proporciones sigan aumentando en los próximos años. Sin embargo, las plantaciones forestales, cuyo objetivo principal sea la rehabilitación ecológica, pueden no siempre ser la mejor estrategia. Lo anterior se debe, en parte, al alto costo y al poco éxito en los procesos de producción y establecimiento de las plántulas (Jordan y Farnworth, 1982; Lamb et al., 2005), así como a la usual baja diversidad genética de las cohortes de plántulas producidas debido, principalmente, a que las semillas se colectan generalmente en una o pocas áreas. Es por esto que es importante determinar opciones que permitan la rehabilitación de los ecosistemas forestales a bajo costo.

La regeneración natural es una opción adecuada para la rehabilitación ecológica de los bosques (Jordan y Farnworth, 1982; Prach y Hobbs, 2008). No obstante, pocos estudios han comparado la estructura de las plantaciones forestales y de la regeneración natural en regiones templadas (p. ej., Bock et al., 1978; Rebottaro y Cabrelli, 2007; Otto et al., 2012; Fernández-Pérez et al., 2013). Para entender la estructura y dinámica de la vegetación, el crecimiento y la densidad en las fases de plántula y juvenil de los árboles son cruciales (Grime y Hillier, 2000), los cuales pueden ser estimadores del desempeño de la rehabilitación.

Por otro lado, la actividad ganadera extensiva provoca cambios en la estructura de tamaños y composición, así como en la densidad de las comunidades arbóreas juveniles (Fleischner, 1994; Milchunas y Lauenroth, 1993; Hernández et al., 2000; Montero-Solís et al., 2006). Sin embargo, no parece existir información sobre si la afectación ocurre del mismo modo sobre la regeneración natural y las plantaciones forestales. Además, pocos estudios han evaluado el desempeño de la exclusión del ganado con un cerco para facilitar el desarrollo de los árboles juveniles (Hernández et al., 2000; Spooner et al., 2002; Spooner y Briggs, 2008). El objetivo de este estudio fue comparar el sistema de reforestación oficial vs. la regeneración natural, específicamente: comparar el número de individuos por hectárea y la tasa anual de crecimiento entre árboles de pinos en edad juvenil, procedentes de las plantaciones forestales y de la regeneración natural, en sitios con diferentes condiciones de reforestación (con y sin reforestación) y exclusión (con y sin ganado vacuno).

\section{Materiales y métodos}

Sitios de estudio y diseño experimental. Se localizaron ocho sitios adyacentes a fragmentos de bosques de pino, previamente deforestados por incendios ocurridos entre ocho y diez años atrás, con diferentes condiciones de regeneración, reforestación y exclusión del ganado vacuno. Todos los sitios se encuentran en los municipios de Las Vigas de Ramírez y Acajete del Estado de Veracruz, México, en la región del Cofre de Perote. Se encuentran a una altitud entre 2,450 y $3,040 \mathrm{~m}$, y presentan condiciones edáficas y climáticas similares: andosoles y climas semifrío subhúmedo y templado húmedo (INEGI, 2007). La vegetación predominante es el bosque de pino (Pinus montezumae, P. patula, P. pseudostrobus (sensu lato) y $P$. teocote; Narave, 1985) con manchones de ilite (Alnus acuminata) y encino (Quercus sp.). Los sitios se escogieron de acuerdo a las siguientes características (Condición): (1) con y sin reforestación, (2) con y sin exclusión de ganado, (3) combinaciones de 1 y 2 . La condición de exclusión de ganado por cercado de púas data desde ocho a diez años previo al registro de este estudio.

Una vez delimitado cada sitio, se escogieron diez puntos aleatorios a lo largo y ancho de cada sitio (con la ayuda de un plano hecho en campo). En cada punto se estableció un cuadro de $100 \mathrm{~m}^{2}$. En cada cuadro se registraron, para cada árbol del género Pinus con altura total entre 15 y 150 $\mathrm{cm}$ (equivalentes a un estadio juvenil), los siguientes datos: especie, origen (Origen: regeneración natural o plantación forestal), altura total $(A L T: \mathrm{cm})$, diámetro basal del tallo $(D B: \mathrm{cm})$, diámetro promedio de cobertura $(C O B: \mathrm{cm})$ y número de verticilos. Para determinar el origen de los árboles (Origen) se consideró: (1) para el caso de la plantación, la presencia de "cajetes" y la distribución espacial regular; y (2) para la regeneración, la ausencia de estos y la distribución espacial aleatoria de los individuos. En campo fue fácil determinar el origen y todos los sitios estaban rodeados de bosque natural.

Análisis de datos. Se calcularon las tasas anuales de crecimiento para cada individuo mediante el enfoque clásico de análisis de crecimiento (Evans, 1972; Hunt, 1990), del siguiente modo:

$$
\Delta_{A L T, C O B}=\left(\frac{\operatorname{In}(V c)}{N v}\right) \text { y } \Delta_{D B}=\left(\frac{\operatorname{In}(V c)+1}{N v+1}\right)
$$

donde, $\Delta_{A L T C O B}$ es la tasa logarítmica promedio del crecimiento relativo anual de altura $A L T$ o diámetro de cobertura $C O B$; para el caso del diámetro de la base del tallo DB se estimó con $\Delta_{D B}$; es decir, se adicionó a cada valor un 1 con la finalidad de no tener logaritmos negativos; $V c$ es la variable morfológica analizada (altura, diámetro basal del tallo o diámetro promedio de cobertura), y $N v$ es el número de verticilos, al cual se le sumó una unidad para representar la edad del individuo expresada en años. El conteo de los verticilos en coníferas como estimador de su edad ha sido documentado en algunos estudios (Niklasson, 2002; Pfeffer, 2005). Para confirmar este supuesto, se seleccionó al azar nueve individuos, los cuales fueron extraídos y llevados al laboratorio para el conteo del número de anillos y verticilos, posteriormente se realizó una correlación de Spearman con el programa R. El número de individuos establecidos por hectárea se estimó a partir de una extrapolación del número de individuos encontrados en la superficie 
muestreada. Se consideró como establecido todo pino con una altura mayor a $15 \mathrm{~cm}$.

El diseño semi-experimental fue no balanceado e incompleto, por lo que se excluyeron de los análisis estadísticos aquellas celdas con ceros estructurales y muestrales (Cuadro 1). Debido a esto, el número de individuos establecidos por hectárea se comparó entre factores con un modelo loglineal, no saturado y anidado, seguido de una estimación de mínimos cuadrados de máxima verosimilitud (proceso CATMOD de SAS 9.2; SAS Institute Inc., 2009); el modelo incluyó la Condición (con y sin reforestación, con y sin exclusión de ganado y, combinaciones de ambos casos), y el Origen (regeneración natural o plantación), el cual se anidó dentro de la Condición.

Para comparar las tasas anuales de crecimiento $\left(\Delta_{A L T}\right.$ ${ }_{D B, C O B}$ ) entre factores, se utilizó un modelo lineal generalizado no saturado, anidado y univariado, seguido de comparaciones múltiples con ajuste de Bonferroni (proceso GLM de SAS 9.2; SAS Institute Inc., 2009). El modelo usado fue:

$$
T C=\text { Cond }+\operatorname{Origen}(\text { Cond })
$$

donde, $T C$ es la tasa de crecimiento (altura, diámetro a la base o la cobertura; según el caso), Cond es el factor Condición (con y sin reforestación, con y sin exclusión de ganado, y, combinaciones de ambos casos), y Origen se refiere al tipo de planta (regeneración natural o plantación). Nótese que el origen está anidado en la condición.

\section{Resultados}

El número de individuos establecidos por hectárea entre la regeneración natural y las plantaciones forestales en sitios

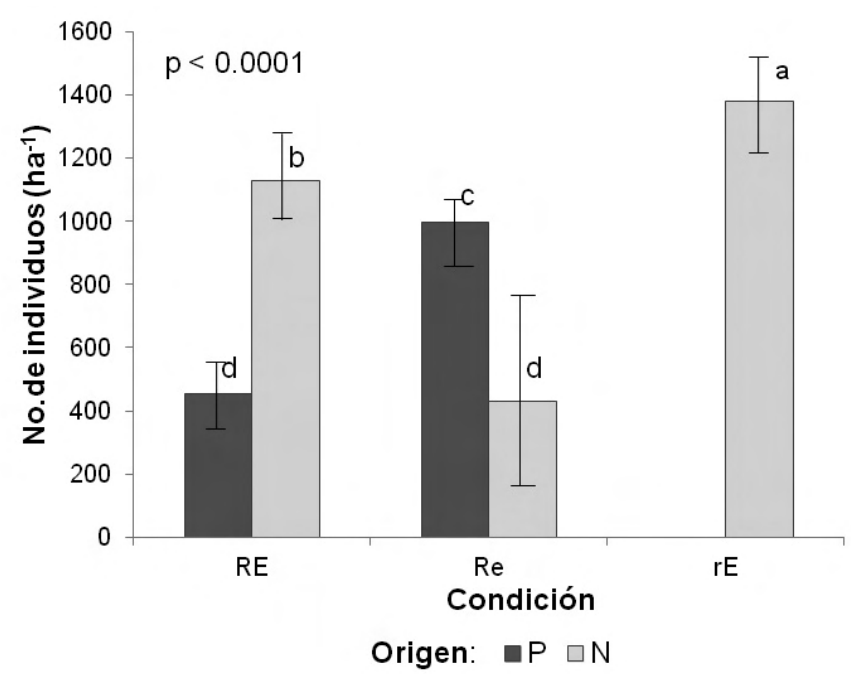

Figura 1. Número de individuos por hectárea y factor de Condición (tratamiento): R, sitio reforestado; E, protegido del ganado; e, sitio no protegido del ganado; r, sitio no reforestado. Factor de Origen: $\mathrm{P}$, plantaciones forestales; $\mathrm{N}$, regeneración natural. Letras diferentes indican diferencias significativas. En el análisis estadístico se usó el número absoluto (frecuencia registrada en el muestreo).
Cuadro 1. Diseño semi-experimental con anidamiento de Condición (Cond) y Origen. $R$, reforestado (ambos, plantación y regeneración natural) y $N r$, no reforestado (únicamente regeneración natural); $E$, con exclusión (presencia de cerco de alambre) y $S e$, sin exclusión (ausencia de cerco de alambre); $P$, plantación y $R n$, regeneración natural. ${ }^{1}=$ celda con cero estructural: el diseño no permite que existan tales individuos ${ }^{2}=$ celda con cero muestral: solamente se registró un individuo para tal condición.

\begin{tabular}{|c|c|c|c|c|c|}
\hline & & \multicolumn{4}{|c|}{ Cond } \\
\hline & & \multicolumn{2}{|c|}{$R$} & \multicolumn{2}{|c|}{$\mathrm{Nr}$} \\
\hline & & $E$ & Se & $E$ & Se \\
\hline \multirow[t]{2}{*}{ Origen } & $P$ & $R E[P]$ & $R S e[P]$ & 1 & 1 \\
\hline & $R n$ & $R E[R n]$ & $R S e[R n]$ & $N r E[R n]$ & 2 \\
\hline
\end{tabular}

con y sin exclusión del ganado fue significativamente diferente $\left(G^{2}=222.06, P<0.0001\right)$. En los sitios protegidos del ganado ( $R E$ y $r E$ ) hubo un mayor número de individuos por hectárea en los de regeneración natural que en las plantaciones forestales; lo contrario ocurrió en los sitios no protegidos del ganado ( $R e$; Figura 1). La regeneración natural en los sitios protegidos del ganado y sin reforestación tuvo el mayor número de individuos por hectárea (Figura 1).

La relación entre número de anillos de crecimiento y número de verticilos fue $r s=0.91, P<0.01$. Las tasas de crecimiento anual de altura promedio $\left(\Delta_{A L T}\right)$, diámetro basal del tallo $\left(\Delta_{D B}\right)$ y diámetro promedio de cobertura $\left(\Delta_{C O B}\right)$ fueron significativamente diferentes entre la regeneración natural y las plantaciones forestales en sitios con y sin ganado $\left(F_{(2,2)}=58.07, P<0.0001 ; F_{(2,2)}=58.6, P<0.0001 ; F_{(2,2)}=\right.$ 62.31. $P<0.0001$; respectivamente). Las mayores tasas de crecimiento anual de altura total y de diámetro promedio de cobertura se registraron en la regeneración natural de los sitios no protegidos del ganado (Re; Figura 2); el crecimiento anual del diámetro basal fue significativamente mayor en la regeneración natural de los sitios reforestados ( $R E$ y $R e$; Figura 2). Las tasas de crecimiento anual de altura total, diámetro basal y diámetro promedio de cobertura más bajas se registraron en las plantaciones forestales de los sitios no protegidos del ganado $(R e)$. La regeneración natural de los sitios no reforestados y protegidos del ganado $(r E)$ tuvieron tasas de crecimiento anual similares a las plantaciones forestales de los sitios protegidos del ganado (RE; Figura 2).

\section{Discusión}

Los resultados de este estudio sugieren que la no exclusión del ganado reduce el número de individuos procedentes de la regeneración natural, en comparación a los sitios con exclusión de ganado (con y sin plantaciones); esto es, que la actividad ganadera es un factor inhibidor del número de individuos que puedan establecerse a partir de la regeneración natural (Fleischner, 1994; Hernández et al., 2000; Montero-Solís et al., 2006). Es también posible que sin ac- 


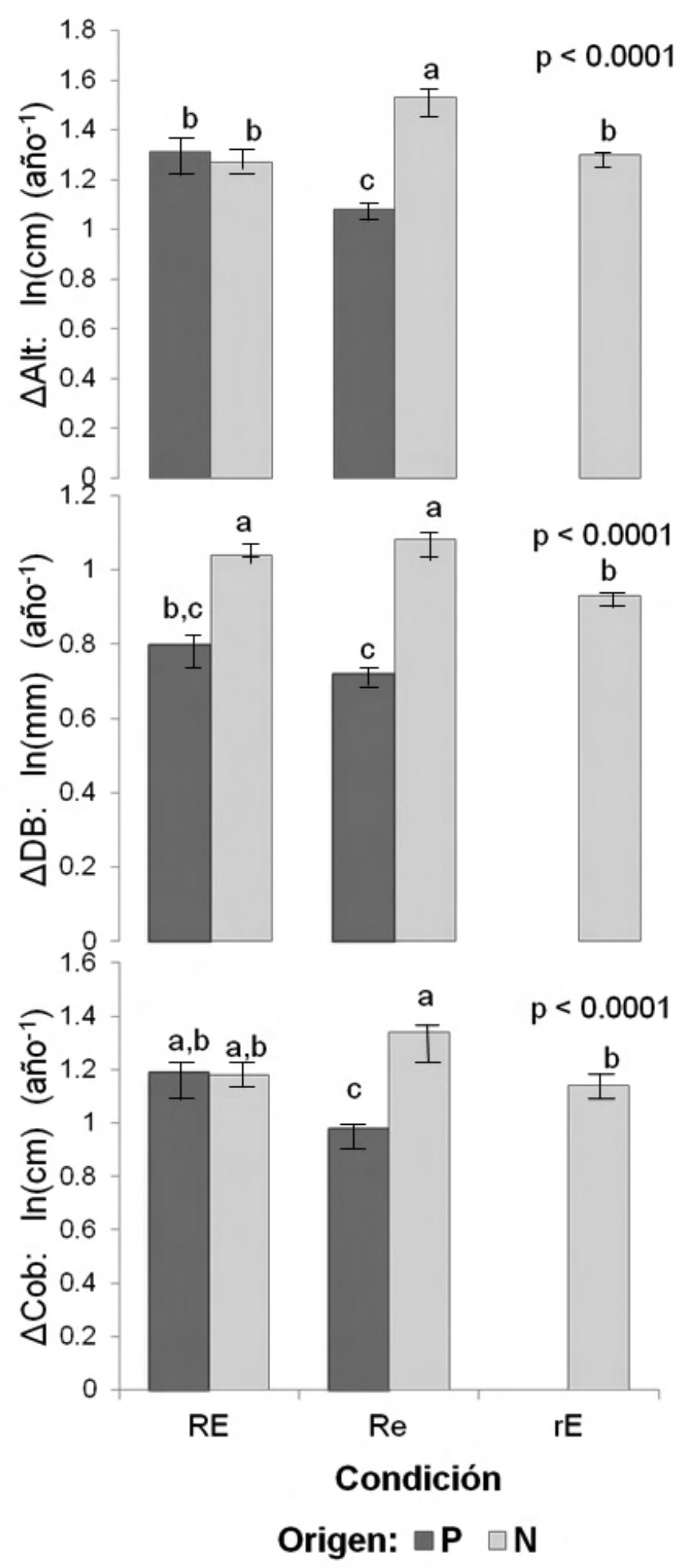

Figura 2. Tasas de crecimiento anual promedio (ln) de la altura $\left(\Delta_{\text {Alt }}\right)$, del diámetro basal del tallo $\left(\Delta_{\mathrm{DB}}\right)$ y del diámetro de cobertura $\left(\Delta_{\mathrm{Cob}}\right)$ por factor de Condición (tratamiento): R, sitio reforestado, E, protegido del ganado; e, sitio no protegido del ganado; r, sitio no reforestado. Factores de Origen: P, plantaciones forestales; N, regeneración natural. Las letras distintas indican diferencias significativas. Las barras indican la desviación estándar de la media. tividad ganadera, como factor de presión, los individuos de regeneración natural estén mejor adaptados a las condiciones microambientales de los sitios que los árboles procedentes de plantación, lo que resulta en una mayor sobrevivencia y establecimiento (p.ej., Hernández et al., 2000). No obstante, los efectos de la exclusión del ganado sobre las plantaciones forestales fueron contrarios; es decir, se encontraron más individuos plantados que de la regeneración natural en los sitios sin exclusión de ganado. Esto sugiere que el efecto negativo de la ganadería sobre el establecimiento de plántulas de la regeneración natural no sólo se debe al ramoneo, sino también a otros factores tales como la compactación del suelo o una menor humedad y mayor temperatura del suelo derivadas de la remoción de la capa de hierbas, las cuales se ha mostrado que afectan negativamente la germinación de semillas (Holl, 1999; Spooner et al., 2002; Hooper et al., 2005).

Los árboles de la regeneración natural presentaron incrementos anuales de altura, diámetro basal y cobertura similares o mayores en los sitios no protegidos del ganado que en aquellos protegidos y, que las plantaciones con y sin exclusión. Esto sugiere que, si bien la actividad ganadera (ganado vacuno) tiene un efecto negativo sobre el número de individuos de regeneración natural que logran establecerse, el efecto sobre su crecimiento parece no ser afectado, contrario a lo ocurrido con los árboles de plantación. Esto podría deberse a la presencia de otras especies que pudieran ser más palatables para el ganado o porque el ganado reduce la densidad de pastos y otras hierbas que compiten con las plántulas de coníferas (Belsky y Blumenthal, 1997); sin embargo, deberá ser evaluado.

La exclusión del ganado bovino, mediante cercos de alambre, en sitios afectados por incendios forestales y cercanos a fuentes de semillas, parece ser una práctica de intervención mínima de bajo costo y adecuada para el establecimiento natural y crecimiento moderado de los individuos. Bajo estas condiciones, puede esperarse un número de individuos establecidos por hectárea similar o mayor a los resultados de una plantación protegida o no protegida del ganado y, mayor que los resultados de un modelo de nula intervención de los sitios. Esto concuerda con las propuestas de algunos autores (Jordan y Farnworth, 1982; Spooner et al., 2002; Hooper et al., 2005; Prach y Hobbs, 2008).

Cabe considerar que, dependiendo del nivel de degradación de los sitios deforestados y de la cercanía a los árboles semilleros, pueden ser necesarias otras prácticas de manejo, tales como la quema y aclareo, para facilitar la regeneración natural (Curtin, 2002; Bailey y Covington, 2002; Rebottaro y Cabrelli, 2007), o el uso de plantaciones sucesionales (Lugo, 1992; Parrota, 1992; Yirdaw, 2002; Sánchez-Velásquez et al., 2009). Por lo tanto, lo más adecuado sería, antes de realizar alguna acción de restauración de un sitio degradado, efectuar una evaluación básica de las características del disturbio que ha ocasionado la degradación, del estado 
actual del sitio, de posibles disturbios futuros y de posibles factores que impidan o dificulten la regeneración natural (Prach y Hobbs, 2008).

En conclusión, la regeneración natural de pinos es una opción adecuada y de bajo costo a las plantaciones forestales en el ámbito de la rehabilitación de sitios degradados por incendios forestales, siempre que sea protegida del ganado y que los terrenos degradados se encuentren cercanos a fragmentos de bosque con árboles semilleros. La regeneración natural protegida del ganado puede tener un crecimiento anual y un número de individuos establecidos por hectárea similares o mayores que las plantaciones forestales.

\section{Agradecimientos}

Este trabajo se realizó dentro de la Línea de Generación y Aplicación del Conocimiento "Ecología Vegetal y Servicios Ecosistémicos" con financiamiento de los fondos SEP-CONACYT Ref. 083060, el Programa de Mejoramiento del Profesorado (PROMEP) de la Secretaría de Educación Pública, el fondo CONACYT SNI-Estudiantes Clave 104598 para tutoría de estudiantes de licenciatura y una beca otorgada por la Universidad Veracruzana para estudiantes de investigadores SNI 1. Los autores agradecen las observaciones y sugerencias hechas por dos revisores anónimos.

\section{Literatura citada}

Bailey J.D. y Covington W.W. 2002. Evaluating ponderosa pine regeneration rates following ecological restoration treatments in northern Arizona, USA. Forest Ecology and Management 155:271-278.

Belsky A.J. y Blumenthal D.M. 1997. Effects of livestock grazing on stand dynamics and soils in upland forests of the Interior West. Conservation Biology 11:315-327.

Bock J.H., Raphael M. y Bock C.E. 1978. A comparison of planting and natural succession after a forest fire in the northern Sierra Nevada. Journal of Applied Ecology 15:597-602.

Curtin C.G. 2002. Livestock grazing, rest, and restoration in arid landscapes. Conservation Biology 16:840-842.

Evans G.C. 1972. The Quantitative Analysis of Plant Growth. University of California Press, Berkeley.

FAO. Organización de las Naciones Unidas para la Agricultura y la Alimentación. 2010. Evaluación de los recursos forestales mundiales 2010. Reporte principal. Estudio FAO: Montes No. 163. Organización de las Naciones Unidas para la Agricultura y la Alimentación, Roma.

FAO. Organización de las Naciones Unidas para la Agricultura y la Alimentación. 2011. Situación de los bosques del mundo 2011. Organización de las Naciones Unidas para la Agricultura y la Alimentación, Roma.

Fernández-Pérez L., Ramírez-Marcial N. y González-Espinosa M. 2013. Reforestación con Cupressus lusitanica y su influencia en la diversidad del bosque de pino-encino en os Altos de Chiapas, México. Botanical Sciences 91:207-216.

Fleischner T.L. 1994. Ecological costs of livestock grazing in Western North America. Conservation Biology 8:629-644.
Grime J.P. y Hillier S.H. 2000. The contribution of seedling regeneration to the structure and dynamics of plant communities, ecosystems and larger units in the landscape. En: Fenner M. Ed. Seeds: The Ecology of Regeneration in Plant Communities, $2^{\mathrm{a}}$ ed., pp. 361-374, CAB International, Wallingford.

Hernández V.G., SánchezV.L.R., Carmona V.T.F., Pineda L.M.R. y Cuevas G.R. 2000. Efecto de la ganadería extensiva sobre la regeneración arbórea de los bosques de la Sierra de Manantlán. Madera y Bosques 6:13-28.

Holl K.D. 1999. Factors limiting tropical rain forest regeneration in abandoned pasture: seed rain, seed germination, microclimate, and soil. Biotropica 31:229-242.

Hooper E., Legendre P. y Condit R. 2005. Barriers to forest regeneration of deforested and abandoned land in Panama. Journal of Applied Ecology 42:1165-1174.

Hunt R. 1990. Basic Growth Analysis. Unwin Hyman, Londres.

INEGI. Instituto Nacional de Estadística y Geografía. 2007. Mapa digital de México v3.0. <http://gaia.inegi.org.mx/mdm6/> (consultado 3 de julio 2013).

Jordan C.F. y Farnworth E.G. 1982. Natural vs. plantation forests: A case study of land reclamation strategies for the humid tropics. Environmental Management 6:485-492.

Lamb D., Erskine P.D. y Parrota J.A. 2005. Restoration of degraded tropical forest landscapes. Science 310:1628-1632.

Lugo A.E. 1992. Tree plantations for rehabilitating damaged forest lands in the tropics. En: Wali M.K. Ed. Ecosystem Rehabilitation: ecosystem analysis and synthesis, pp. 247-255, SPB Academic Publishing, La Haya.

Michon G. y de Foresta H. 1996. Agroforests as an alternative to pure plantations for the domestication and commercialization of NTFPs. En: Leakey R.R.B., Temu A.B., Melnyk M. y Vantomme P. Eds. Domestication and Commercialization of Nontimber Forest Products in Agroforestry Systems, pp. 160-175, Organización de las Naciones Unidas para la Agricultura y la Alimentación, Roma.

Milchunas D.G. y Lauenroth W.K. 1993. Quantitative effects of grazing on vegetation and soil over a global range of environments. Ecological Monographs 63:327-366.

Montagnini F., Cusack D., Petit B. y Kanninen M. 2004. Environmental services of native tree plantations and agroforestry systems in Central America. Journal of Sustainable Forestry 21:51-67.

Montero-Solís F.M., Sánchez-Velásquez L.R., Pineda-López M.R., Martínez-Rivera L.M., Moermond T. y Corral A.J. 2006. Livestock impact on dynamic and structure of tropical dry forest of the Sierra de Manantlán, Mexico. Journal of Food, Agriculture and Environment 4:266-270.

Narave H. 1985. La vegetación del Cofre de Perote, Veracruz, México. Biótica 10:35-64.

Niklasson M. 2002. A comparison of three age determination methods for suppressed Norway spruce: implications for age structure analysis. Forest Ecology and Management 161:279-288.

Otto R., García-del-Rey E., Méndez J. y Fernández-Palacios J.M. 2012. Effects of thinning on seed rain, regeneration and understory vegetation in a Pinus canariensis plantation (Tenerife, Canary Islands). Forest Ecology and Management 280:71-81.

Parrota J.A. 1992. The role of plantation forests in rehabilitating degraded tropical ecosystems. Agriculture, Ecosystems and Environment 41:115-133. 
Pfeffer M.D. 2005. Regression-based age estimates of yellow pine saplings, Jefferson National Forest, Virginia. Tesis licenciatura, Universidad de Tennessee-Knoxville, Knoxville. 49 pp.

Prach K. y Hobbs R.J. 2008. Spontaneous succession versus technical reclamation in the restoration of disturbed sites. Restoration Ecology 16:363-366.

Rebottaro S.L. y Cabrelli D.A. 2007. Crecimiento y rendimiento comercial de Pinus elliottii en plantación y en regeneración natural manejada con raleos en Entre Ríos, Argentina. Bosque 28:152-161.

Sánchez-Velásquez L.R., Pineda-López M.R., Galindo-González J., Díaz-Fleischer F. y Zúñiga G.J.L. 2009. Opportunity for the study of critical successional processes for the restoration and conservation of mountain forest: the case of Mexican pine plantations. Interciencia 34:518-522.

SAS Institute Inc. 2009. SAS System for windows version 9.2.
SAS Institute Inc., Cary.

Sedjo R.A. 1999. The potential of high-yield forestry for meeting timber needs. New Forests 17:339-360.

Spooner P.G. y Briggs S.V. 2008. Woodlands on farms in southern New South Wales: A longer-term assessment of vegetation changes after fencing. Ecological Management and Restoration 9:33-41.

Spooner P., Lunt I. y Robinson W. 2002. Is fencing enough? The short-term effects of stock exclusion in remnant grassy woodlands in southern NSW. Ecological Management and Restoration 3:117-126.

Yirdaw E. 2002. Restoration of native woody-species diversity, using plantation species as foster trees, in the degraded highlands of Ethiopia. Tesis doctoral, Universidad de Helsinki, Helsinki. $61 \mathrm{pp}$.

Recibido: 19 de agosto de 2013

Aceptado: 4 de noviembre de 2013 